\title{
Minimally invasive fetal surgery for myelomeningocele: preliminary report from a single center
}

\author{
Giorgio Carrabba, MD, PhD, ${ }^{1}$ Francesco Macchini, MD, ${ }^{2}$ Isabella Fabietti, MD, ${ }^{3}$ \\ Luigi Schisano, MD, ${ }^{1}$ Giulia Meccariello, MD, ${ }^{1}$ Rolando Campanella, MD, ${ }^{1}$ \\ Giulio Bertani, MD, PhD, ${ }^{1}$ Marco Locatelli, MD, PhD, ${ }^{1}$ Simona Boito, MD, ${ }^{3}$ \\ Giuliana A. Porro, MD, ${ }^{4}$ Lorenzo Gabetta, MD, ${ }^{5}$ Odoardo Picciolini, MD, ${ }^{6}$ Claudia Cinnante, MD, ${ }^{7}$ \\ Fabio Triulzi, MD, ${ }^{7}$ Fabrizio Ciralli, MD, ${ }^{8}$ Fabio Mosca, MD, ${ }^{8}$ Denise A. Lapa, MD, PhD, ${ }^{9}$ \\ Ernesto Leva, MD, ${ }^{2}$ Paolo Rampini, MD, ${ }^{1}$ and Nicola Persico, MD $^{3}$
}

Departments of ${ }^{1}$ Neurosurgery, ${ }^{2}$ Pediatric Surgery, ${ }^{3}$ Obstetrics—Fetal Surgery, ${ }^{4}$ Anesthesia and Intensive Care, ${ }^{5}$ Pediatric Plastic Surgery, ${ }^{6}$ Pediatric Physiatry, ${ }^{7}$ Neuroradiology, and ${ }^{8}$ Neonatal Intensive Care, Fondazione IRCCS Ca' Granda Ospedale Maggiore Policlinico, Milano, Italy; and ${ }^{9}$ Hospital Israelita Albert Einstein Centro De Terapia Fetal, São Paulo, Brazil

\begin{abstract}
OBJECTIVE Recent trials have shown the safety and benefits of fetoscopic treatment of myelomeningocele (MMC). The authors' aim was to report their preliminary results of prenatal fetoscopic treatment of MMC using a biocellulose patch, focusing on neurological outcomes, fetal and maternal complications, neonatal CSF leakage, postnatal hydrocephalus, and radiological outcomes.
\end{abstract}

METHODS Preoperative assessment included clinical examination, ultrasound imaging, and MRI of the fetus. Patients underwent purely fetoscopic in utero MMC repair, followed by postoperative in utero and postnatal MRI. All participants received multidisciplinary follow-up.

RESULTS Five pregnant women carrying fetuses affected by MMC signed informed consent for the fetoscopic treatment of the defect. The mean MMC size was $30.4 \mathrm{~mm}$ (range 19-49 mm). Defect locations were L1 (2 cases), L5 (2 cases), and L4 (1 case). Hindbrain herniation and ventriculomegaly were documented in all cases. The mean gestational age at surgery was 28.2 weeks (range 27.8-28.8 weeks). Fetoscopic repair was performed in all cases. The mean gestational age at delivery was 33.9 weeks (range 29.3-37.4 weeks). After surgery, reversal of hindbrain herniation was documented in all cases. Three newborns developed signs of hydrocephalus requiring CSF diversion. Neurological outcomes in terms of motor level were favorable in all cases, but a premature newborn died due to CSF infection and sepsis.

CONCLUSIONS The authors' preliminary results suggest that fetoscopic treatment of MMC is feasible, reproducible, and safe for mothers and their babies. Neurological outcomes were favorable and similar to those in the available literature. As known, prematurity was the greatest complication.

https://thejns.org/doi/abs/10.3171/2019.8.FOCUS19438

KEYWORDS myelomeningocele; spina bifida; fetoscopy; hydrocephalus

$\mathrm{M}$ YELOMENINGOCELE (MMC) is an anatomical abnormality caused by a congenital defect of the central nervous system, resulting in the protrusion of meninges and the spinal cord through a defect of the vertebral arch. The radiological presentation is characterized by ventriculomegaly, hindbrain herniation, and syringomyelia. Infants may present with lower-limb sensory and motor defects; bladder, bowel, and erectile dysfunction; and mental retardation. Children affected by MMC require immediate surgical care to treat CSF leakage, prevent infection, and restore, as much as possible, the correct anatomical conformation. Further surgeries are often indicated to treat associated conditions such as hydrocephalus, hindbrain herniation, and tethered cord. ${ }^{4}$ These babies need lifelong multidisciplinary support and follow-up to deal with urological, orthopedic, and neurological issues, which are primarily or secondarily associated with MMC. The severity of this condition was confirmed by a population-

ABBREVIATIONS MMC = myelomeningocele; MOMS = Management of Myelomeningocele Study; PROM = premature rupture of membranes .

SUBMITTED June 1, 2019. ACCEPTED August 6, 2019.

INCLUDE WHEN CITING DOI: 10.3171/2019.8.FOCUS19438. 
based study that estimated the survival rate at 1,10 , and 20 years of age to be $71 \%, 69 \%$, and $66 \%$, respectively. ${ }^{15}$

The rationale of prenatal surgical closure of MMC is to minimize toxic injury to the exposed spinal cord during pregnancy due to amniotic fluid. A randomized controlled study, Management of Myelomeningocele Study (MOMS), was designed to compare the benefits of prenatal repair of the malformation via open fetal surgery with standard postnatal repair. This trial demonstrated a potential role of prenatal surgery in improving neurological outcomes and preventing neurological complications such as hydrocephalus and hindbrain herniation. Indeed, the incidence of hydrocephalus decreased from $82 \%$ in the postnatal repair group to $40 \%$ in the prenatal surgical group. ${ }^{2}$ The trial, which was interrupted early because of the efficacy of prenatal surgery, also documented an increase in maternal morbidity in comparison with postnatal surgery, with the most common complication being oligohydramnios, placental abruption, premature rupture of membranes (PROM), preterm delivery, and uterine scar dehiscence.

To minimize consequences to the mother and fetus, minimally invasive endoscopic approaches have been proposed. Kohl et al. and Pedreira et al. described different minimally invasive fetoscopic techniques with promising results. ${ }^{8,10}$ In fact, the improvements in terms of neurological outcomes and reversal of hindbrain herniation and hydrocephalus were similar to those in the MOMS trial. Moreover, maternal morbidity and fetal mortality were significantly reduced by these techniques. The major drawbacks of the fetoscopic correction were the prematurity and the complexity of the surgical technique..$^{5-7}$

The aim of this work was to describe our preliminary experience with the fetoscopic treatment of MMC in terms of neurological outcomes, fetal and maternal complications, neonatal CSF leakage, postnatal hydrocephalus, and radiological outcomes.

\section{Methods \\ Patients}

The study was approved by the Fondazione IRCCS Ca' Granda Ospedale Maggiore Policlinico ethics committee.

A team comprising fetal surgeons/gynecologists, pediatric surgeons, pediatric intensive care specialists, and neurosurgeons underwent specific training at the beginning of 2018. The training consisted of visiting the Hospital Israelita Albert Einstein of São Paulo twice (1 case of fetoscopic treatment with a posterior placenta and 1 case of an anterior placenta). Two cases were arranged at our institution with the support of the entire Brazilian team. The following cases were managed locally with the presence of the Brazilian senior surgeon.

All patients referred to Fondazione IRCCS Ca' Granda Ospedale Maggiore Policlinico with a prenatal diagnosis of MMC underwent multidisciplinary maternal counseling. Preoperative assessment included physical examination, ultrasound, and MRI.

Inclusion and exclusion criteria are summarized in Table 1. Briefly, the inclusion criteria were similar to the ones selected in the MOMS trial: single pregnancy, myelomeningocele at level T1 through S1, evidence of hindbrain
TABLE 1. Inclusion and exclusion criteria for fetoscopic repair of open spina bifida

\begin{tabular}{ll}
\hline \multicolumn{1}{c}{ Inclusion Criteria } & \multicolumn{1}{c}{ Exclusion Criteria } \\
\hline Myelomeningocele T1-S1 & Multiple gestation \\
\hline Hindbrain herniation & Kyphosis $>30^{\circ}$ \\
\hline Maternal age $>18$ yrs & Cervical length $<2 \mathrm{~cm}$ \\
\hline No other major anomalies & Low-lying placenta \\
\hline Normal karyotype & Placental abruption \\
\hline & History of preterm birth $<37$ wks \\
\hline & History of incompetent cervix \\
\hline & Alloimmunization \\
\hline & Positive serology (HIV, hepatitis B) \\
\hline & Large uterine fibroids \\
\hline & BMI $>35$ kg/m ${ }^{2}$ \\
& Maternal conditions that may significantly \\
& increase the risk of preterm delivery, \\
& uterine bleeding, or serious anesthe- \\
& sia-related complications \\
\hline
\end{tabular}

herniation, a normal karyotype, maternal age of at least 18 years, and consent from both parents. Exclusion criteria were major fetal abnormalities unrelated to the MMC, risk of preterm birth (e.g., previous preterm birth), and contraindication to surgery such as previous hysterotomy.

The following data were collected prospectively and included before surgery: maternal age, parity, placenta position, upper level of the lesion, lesion size, presence of hindbrain herniation, ventricular dilatation, syringomyelia, and fetal leg movements. Furthermore, we evaluated gestational age at surgery; surgical technique; number of patches needed; skin-to-skin operative time; and intraoperative and postoperative complications, including gas embolism, pulmonary edema, need for blood transfusion, chorioamnionitis, uterine dehiscence, interval between surgery and PROM, and delivery mode.

In addition to preoperative MRI, postoperative in utero MRI was performed; after birth, additional brain and spinal cord MRI scans were performed. The following parameters were investigated on MRI: site and extent of the defect, hindbrain herniation, hydrocephalus, and other malformations.

Collected data at birth included gestational age at birth; birth weight; prematurity-related complications; presence of CSF leakage, infection, fistula, or skin dehiscence requiring surgical repair; seizures; reversal of hindbrain herniation; need for a CSF shunt; and birth-to-CSF shunting interval.

All patients were finally referred for multidisciplinary follow-up to evaluate maternal morbidity and the newborn's neurological outcome. In particular, neurological evaluation was assessed, comparing the functional sensory/motor deficit to the anatomical level of the lesion, which was calculated using postdelivery MRI.

\section{Surgical Technique}

Surgery was performed under total intravenous anesthesia. Neither direct fetal anesthesia nor intraoperative 
tocolytics were used. An ultrasound-guided amnioinfusion of $500 \mathrm{~mL}$ warm ringer lactate was performed to gain enough room in the amniotic cavity to allow safer trocar insertion. Then, the percutaneous Seldinger technique under ultrasound control was used to access the uterine cavity for the placement of 4 trocars: one 5-mm balloontipped laparoscopic trocar and three $11-\mathrm{Fr}$ vascular introducers. The position of trocars changed according to the position of the placenta. For a posterior placenta, trocars were inserted at the level of the lower uterine wall; for an anterior placenta, they were positioned in the uterine side according to the course of the main vessels.

After the removal of amniotic fluid, $\mathrm{CO}_{2}$ insufflation was started, and the initial intrauterine pressure was measured. Humidification and heating of the $\mathrm{CO}_{2}$ (Lexion Medical) was used in all cases. The upper pressure limit was set at 3-5 $\mathrm{mm} \mathrm{Hg}$ above the initial uterine pressure, which was on average $14 \mathrm{~mm} \mathrm{Hg}$ (range $10-18 \mathrm{~mm} \mathrm{Hg}$ ), and gas flow was set at $15 \mathrm{~L} / \mathrm{min}$.

The fetus was positioned using standard 3.5-mm laparoscopic instruments (Aesculap) under fetoscopic control using a 4-mm $30^{\circ}$ endoscope (Aesculap). The position of the fetus is crucial to make the repair easier and safer. The ideal position of the fetus is with the head facing down toward the surgeon and the buttocks facing up, opposite to the surgeon, so that the spine is in line with the camera (Fig. 1A). The neural placode was released using a circumferential incision at the transition zone (Fig. 1B). The skin was further undermined to allow approximation of the edges in the midline. After assessing the feasibility of primary skin closure, a biocellulose patch (Bionext) was fashioned and positioned over the placode (Fig. 1C). The skin was then closed over the patch with a single running suture. We used a 2-0 self-blocking nonabsorbable monofilament stitch (polypropylene).

In cases in which the skin defect was too large to allow primary skin approximation, 2 patches were used: a bilaminar skin substitute was placed on top of the biocellulose patch. The biocellulose patch was secured to the bilaminar skin substitute by placing 1 or 2 separated single sutures prior to their insertion through the trocar. This was done to ensure that the 2 layers would not slide past each other. The skin substitute with the attached biocellulose was then placed over the defect with the silicone layer facing up (exposed to the amniotic fluid). Special care was taken to ensure that the patch would remain under the edges of the undermined skin. This was accomplished by using 2 running 2-0 self-blocking monofilament stitches. The size of the biocellulose patch was determined to be the small- est diameter needed to cover the dura mater gap, and the bilaminar substitute was cut $0.5 \mathrm{~cm}$ larger than the biocellulose patch. The trocars were then removed with no need for uterine closure devices to seal the puncture sites.

After birth, the bilaminar patch was kept in place and protected using a wound dressing. These dressings have borders that are firmly adherent to the neonatal skin. The purpose of these dressings was to keep the lesion clean and free of feces and urine. Sutures were removed after spontaneous detachment of the silicone layer from the dermal matrix was noted. The dressing was changed at least every 2-3 days, until complete skin healing was achieved.

\section{Results}

From June 2018 to December 2018, 5 patients underwent fetoscopic repair of MMC. At the prenatal assessment, the upper level of the lesion was L1 and L5 in 2 cases each and L4 in 1 case. The mean defect length was 30.36 $\mathrm{mm}$ (range 19-49 $\mathrm{mm}$ ), while the mean width was $23 \mathrm{~mm}$ (range 12-42 $\mathrm{mm}$ ). Placenta position was posterior in 3 cases and anterior in 2 cases (Table 2). Preoperative MRI (Fig. 2) showed signs of hindbrain herniation and ventriculomegaly (mean ventricle size $13.62 \mathrm{~mm}$ ) in all cases. Syringomyelia was present in only 1 case.

At surgery, the mean maternal age was 35 years (range $22-43$ years). Two mothers were nulliparous, and 3 were multiparous (parity 2). The mean gestational age at surgery was 28.2 weeks (range 27.8-28.8 weeks). Fetoscopy was performed and completed in all cases; the mean skinto-skin operative time was 204 minutes (range 140-270 minutes). In 4 patients, a single-layer technique with a biocellulose patch was performed (Fig. 1), whereas in 1 case a large $(49 \times 42-\mathrm{mm})$ defect was repaired with a 2-layer technique. No intraoperative complication was recorded; in particular, there was no evidence of gas embolism or pulmonary edema, and no blood transfusion was required. No patient required admission to the ICU, and all mothers and fetuses survived (Table 3).

PROM occurred in 4 cases at a mean gestational age of 31.8 weeks (range 29.1-35.1 weeks). The median interval between surgery and PROM was 3.3 weeks (range 1.3-7.3 weeks). Delivery was cesarean in 3 cases and vaginal in 2 cases. The median gestational age at delivery was 35.8 weeks (range 29.3-37.4 weeks). The mean interval from operation to delivery was 3.8 weeks (range 1.3-8.6 weeks). There were no placental abruptions, preeclampsia, or uterine dehiscence, and a blood transfusion was not needed. One patient developed a chorioamnionitis and was treated

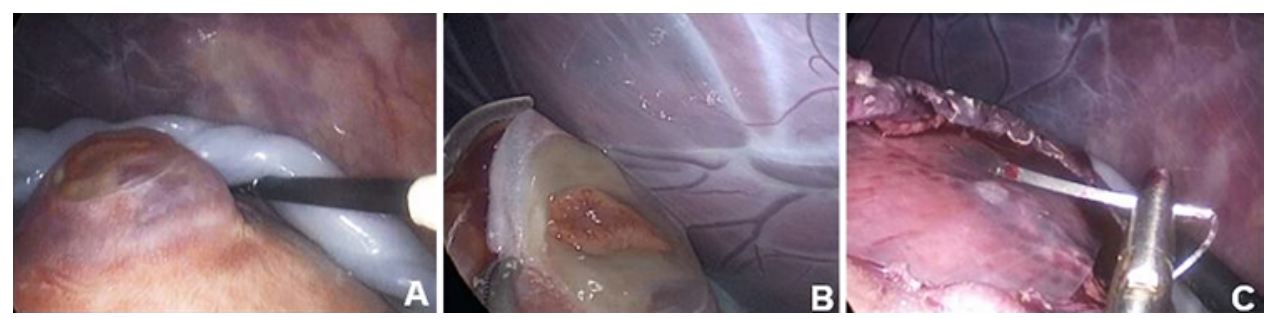

FIG. 1. Steps of in utero MMC repair. A: Intraoperative fetosopic view of an intact MMC; the placode is clearly visible. B: Placode release at the transitional zone. C: Placement of the biocellulose patch over the defect. 
TABLE 2. Preoperative assessment data

\begin{tabular}{lccccc}
\hline & Case 1 & Case 2 & Case 3 & Case 4 & Case 5 \\
\hline Placental position & Posterior & Anterior & Anterior & Posterior & Posterior \\
\hline Defect & MMC & MMC & MMC & MMC & MMC \\
\hline Upper lesion level & L1 & L1 & L5 & L5 & L4 \\
\hline Lesion length, mm & 49 & 27.8 & 19 & 30 & 26 \\
\hline Lesion width, mm & 42 & 12 & 14 & 27 & 20 \\
\hline $\begin{array}{c}\text { Largest ventricle, } \\
\text { mm }\end{array}$ & 13.8 & 16.1 & 9 & 12.2 & 17 \\
\hline
\end{tabular}

Imaging studies with ultrasonography and MRI were used to evaluate placental position, type of defect, as well as site and measurements of the lesion and the associated ventriculomegaly.

subsequently with no major consequences. The mean birth weight was $2049 \mathrm{~g}$ (range 1400-2550 g).

CSF leak was noted at birth in 1 patient (born 10 days after in utero correction of the defect). Unfortunately, CSF infection occurred (Escherichia coli) and was complicated by cerebral abscesses and sepsis, resulting in death of the baby (> 28 days).

Three babies developed partial skin dehiscence after birth (without CSF leakage) requiring surgical revision, which was performed together with a pediatric plastic surgeon between 9 and 108 days after birth.

In utero postoperative MRI could be performed in 3 fetuses at a mean gestational age of 29 weeks and, interestingly, the examination showed a complete reversal of hindbrain herniation in all cases (Fig. 3).

MRI was performed in all newborns and confirmed a complete reversal of hindbrain herniation in 3 cases and a partial reversal in 2 cases. No CNS injuries (e.g., intraventricular hematoma, periventricular leukomalacia) were noted on postnatal MRI.

Regarding motor outcomes, at birth, in 4 cases the functional motor level was lower than the upper anatomical level of the defect; in 1 case the anatomical and functional motor levels were the same (Table 4).

Three newborns developed signs of hydrocephalus and required a ventriculoperitoneal shunt ( 2 cases) or ventricular catheter with reservoir (1 case) (Table 4). In all 3 cases, signs of increased intracranial pressure, such as bulging fontanelle, split sutures, and increase in the head circum-

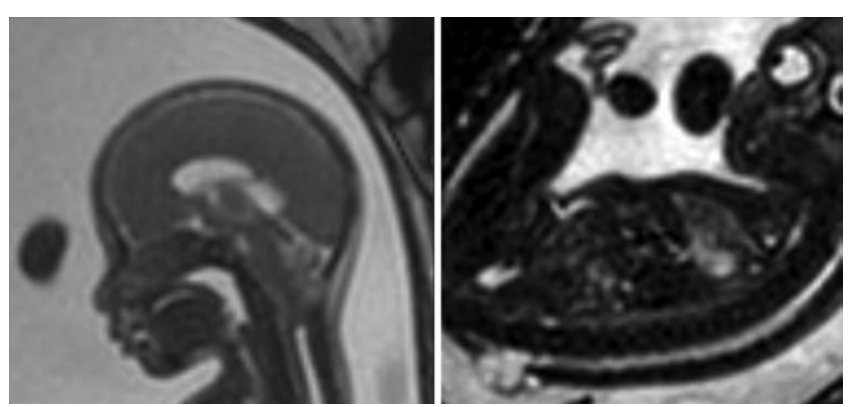

FIG. 2. Sagittal MRI sequences obtained in a fetus at 21.1-weeks' gestation, documenting Chiari II malformation with a small posterior fossa, low attachment of the tentorium, and hindbrain herniation (left) and MMC (right).
TABLE 3. Surgery and delivery data

\begin{tabular}{lccccc}
\hline & Case 1 & Case 2 & Case 3 & Case 4 & Case 5 \\
\hline GA at op, wks & 28.1 & 28.8 & 28.3 & 27.8 & 27.8 \\
\hline No. of patches used & 2 & 1 & 1 & 1 & 1 \\
\hline Length of op, mins & 210 & 270 & 140 & 230 & 170 \\
\hline Gas embolism & No & No & No & No & No \\
\hline Pulmonary edema & No & No & No & No & No \\
\hline Chorioamnionitis & Yes & No & No & No & No \\
\hline GA at PROM, wks & 30.9 & NA & 32.1 & 29.1 & 35.1 \\
\hline Op to PROM, wks & 2.8 & NA & 3.8 & 1.3 & 7.3 \\
\hline GA at delivery, wks & 31.0 & 37.4 & 36.0 & 29.3 & 35.8 \\
\hline Mode of delivery & CS & CS & CS & V & V \\
\hline
\end{tabular}

$\mathrm{CS}=$ cesarean section; $\mathrm{GA}=$ gestational age; $\mathrm{NA}=$ not applicable $\mathrm{V}=$ vaginal delivery.

The table shows data relative to surgeries, associated complications, timing in which PROM occurred relatively to surgery, and delivery method.

ference adjusted for gestational age, led the pediatric neurosurgeon to perform surgery between 14 and 74 days after surgery.

\section{Discussion}

Myelomeningocele is one of the most common congenital malformations of the nervous system, resulting in an incomplete closure of neural tube leading to an extrusion of meninges and the spinal cord in a sac that protrudes through the skin of the thoracolumbar area. The exact etiopathogenetic mechanism remains unclear. In utero deterioration is usually explained by the 2-hit pathogenesis, according to which the neural tube fails to close, exposing nervous structures to the toxic effect of amniotic fluid. Moreover, continuous CSF leakage causes a suction gradient, leading to hindbrain herniation, Chiari malformation, and ventriculomegaly. ${ }^{3}$

Historically, the management of newborns with MMC was based on early postnatal closure of the spinal defect and supportive intervention such as ventriculostomy. ${ }^{1}$ The introduction of better imaging techniques, including highresolution ultrasonography, and ultra-fast MRI, together with fetal karyotyping and maternal serum screening, allowed early prenatal identification of congenital neural tube defects. ${ }^{12,14}$ Large-animal models, such as the lamb model introduced by Meuli et al. in 1995, were developed to evaluate if a prenatal correction could prevent spinal cord deterioration and subsequently ameliorate neurological status at birth., ${ }^{913}$ The classic postnatal surgical management of MMC was challenged by the MOMS study in 2011. ${ }^{2}$ Briefly, the MOMS trial, referring to intrauterine open surgical correction of MMC, showed that prenatal correction resulted in better neurological outcomes at the expense of maternal morbidity when compared with postnatal surgery.

Trying to reproduce the positive results of the MOMS study, the CECAM (Cirurgia Endoscópica para Correção Antenatal da Meningomielocele) study was designed in 2016 aiming to reduce the maternal risk of prenatal surgical management of MMC. In that study, the authors 

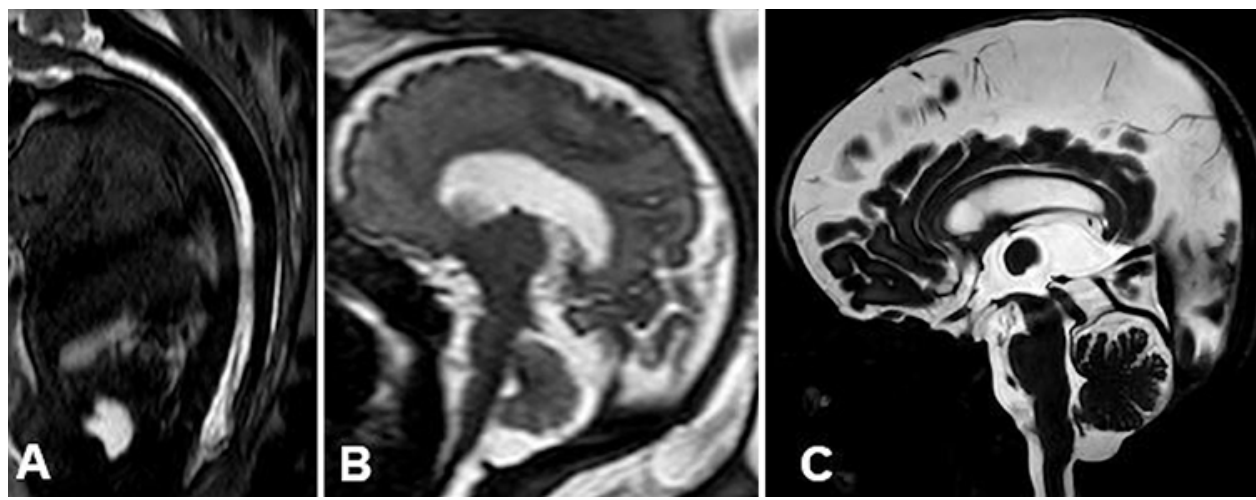

FIG. 3. Postoperative MR images obtained in the case shown in Fig. 1. A and B: Fetal images (gestational age of 33 weeks) documenting MMC correction (A) and total reversal of hindbrain herniation and improved visualization of CSF spaces (B). C: Sagittal midline image obtained at birth, confirming total reversal of hindbrain herniation.

described an in utero fetoscopic technique of MMC closure, which was based on the placement of a biocellulose patch over the defect followed by skin closure with single running stitches. ${ }^{10}$ The trial demonstrated the efficacy of this surgical method in terms of neurological outcomes, reversal of hindbrain herniation, and prevention, or, at least delay, of CSF shunt necessity, without increasing maternal morbidity. The main concerns about the surgical approach were PROM and prematurity, the complexity of the fetoscopic technique, and the higher rate of dehiscence or leakage at the MMC repair site (eventually requiring postnatal revision). ${ }^{5}$

The purpose of this paper was to describe our preliminary experience with the fetoscopic repair of MMC, eventually supporting the results of the CECAM and similar studies in MMC prenatal treatment.

Regarding the surgical technique, after dedicated training (see Methods), we were able to safely perform the purely endoscopic surgical procedure with success in all cases. In fact, the closure was watertight in 4 patients; the only newborn with CSF leakage was born 10 days after the intrauterine correction of the MMC, and the postoperative time was probably not sufficient to obtain complete healing of the wound (which has to happen in the amniotic fluid). At that time, the baby had a gestational age of 29 weeks with a weight of $1400 \mathrm{~g}$; unfortunately, this baby died a few weeks after birth due to CSF infection and sepsis. This case illustrates the well-described severe consequences of prematurity and early birth after intrauterine surgery. In fact, as mentioned in MOMS, potential benefits of prenatal surgery must be balanced against the risks of prematurity and maternal morbidity.

Regarding the effects of the intrauterine fetoscopic correction, our work showed, for the first time, that early changes could be observed on in utero postoperative MRI. In fact, reversal of hindbrain herniation was appreciable in all cases in which postoperative in utero MRI was performed, indicating that the closure of the defect was able to stop the suction gradient, which might be responsible for secondary injuries to the CNS (Fig. 3).

The positive neurological outcomes that we observed, in agreement with the current literature, could be interpreted as a consequence of early correction of the gradient and reduced exposure of the spinal cord to the relative toxicity of the amniotic fluid. ${ }^{6,7}$ In fact, the motor level in most cases ( 4 of 5) was better than the anatomical level of lesion.

Moreover, the complete reversal of hindbrain herniation, which was observed in 3 cases, probably helped avoid the development of symptomatic hydrocephalus in 2 cases. Despite the limited number of patients, this result appears to confirm data in the literature regarding the significant reduction in the need for CSF shunting after intrauterine MMC closure. ${ }^{16}$

Regarding the closure of the skin defects, some authors have argued that the current fetoscopic techniques of a single or double layer are far from meeting neurosurgical standards. ${ }^{11}$ In fact, the optimal reconstruction would consist of a 3-layer repair: the dura mater, the myofascial layer, and the skin. In this series, we used a single-layer closure technique, as described by Pedreira et al., which was effective in terms of watertight closure, reversal of hindbrain herniation, and neurological outcomes. ${ }^{10}$ Nevertheless, 3 babies showed progressive dehiscence of the thoracolumbar wound (without any CSF leakage), which was managed surgically by a plastic surgeon. The other operations were straightforward and uneventful.

These relatively good short-term outcomes need to be confirmed over time, in particular with regard to the late complications of MMC corrections such as tethered cord.

TABLE 4. Postnatal data

\begin{tabular}{lccccc}
\hline & Case 1 & Case 2 & Case 3 & Case 4 & Case 5 \\
\hline Birth weight, g & 1630 & 2270 & 2550 & 1400 & 2395 \\
\hline CSF leakage & No & No & No & Yes & No \\
\hline $\begin{array}{l}\text { Reversal of HH docu- } \\
\text { mented on MRI }\end{array}$ & Partial & Yes & Yes & Partial & Yes \\
\hline $\begin{array}{l}\text { Upper anatomical level } \\
\text { of lesion }\end{array}$ & T12 & L1 & L4 & L4 & L3 \\
\hline Motor level & L4 & L5 & L4 & L5 & L5 \\
\hline CSF shunting & Yes & Yes & No & Yes $^{*}$ & No \\
\hline
\end{tabular}

$\mathrm{HH}=$ hindbrain herniation.

* This patient had a ventricular catheter with reservoir but not a full definitive shunt. 


\section{Conclusions}

Fetal intervention offers the possibility of repairing malformations that are otherwise associated with poor neurological development. Even if we are still at the beginning of our learning curve, the endoscopic approach appeared feasible and safe. The risk of a failed closure and the need for following surgical procedures were observed, but they were overcome by the promising neurological outcome as well as the reversal of hindbrain herniation demonstrated on MRI. Nevertheless, a newborn died of complications related to prematurity, suggesting that potential benefits of prenatal surgery must be balanced against the risks of prematurity. A larger pool case and a longer multidisciplinary follow-up will help us understand to what extent we can recommend the fetoscopic approach.

\section{Acknowledgments}

We are really grateful to Rodrigo T. R. Goncalves, Gregorio L. Acacio, and Vanessa Barba who, through their teaching, permitted the successful accomplishment of these operations. We want to thank for their collaboration and support all the nurses of the Mangiagalli operating room, with special mentions to Carlo Scala, Elisabetta Nervi, and Daniela Accolla. Finally, we want to thank all the mothers and fathers of the babies operated on fetoscopically for their trust in science and innovation.

\section{References}

1. Adzick NS: Fetal surgery for spina bifida: past, present, future. Semin Pediatr Surg 22:10-17, 2013

2. Adzick NS, Thom EA, Spong CY, Brock JW III, Burrows PK, Johnson MP, et al: A randomized trial of prenatal versus postnatal repair of myelomeningocele. N Engl J Med 364:993-1004, 2011

3. Heffez DS, Aryanpur J, Hutchins GM, Freeman JM: The paralysis associated with myelomeningocele: clinical and experimental data implicating a preventable spinal cord injury. Neurosurgery 26:987-992, 1990

4. Heuer GG, Moldenhauer JS, Scott Adzick N: Prenatal surgery for myelomeningocele: review of the literature and future directions. Childs Nerv Syst 33:1149-1155, 2017

5. Kabagambe SK, Jensen GW, Chen YJ, Vanover MA, Farmer DL: Fetal surgery for myelomeningocele: a systematic review and meta-analysis of outcomes in fetoscopic versus open repair. Fetal Diagn Ther 43:161-174, 2018

6. Keller BA, Farmer DL: Fetal surgery for myelomeningocele: history, research, clinical trials, and future directions. Minerva Pediatr 67:341-356, 2015

7. Kitagawa H, Pringle KC: Fetal surgery: a critical review. Pediatr Surg Int 33:421-433, 2017
8. Kohl T, Tchatcheva K, Merz W, Wartenberg HC, Heep A, Müller A, et al: Percutaneous fetoscopic patch closure of human spina bifida aperta: advances in fetal surgical techniques may obviate the need for early postnatal neurosurgical intervention. Surg Endosc 23:890-895, 2009

9. Meuli M, Meuli-Simmen C, Hutchins GM, Yingling CD, Hoffman KM, Harrison MR, et al: In utero surgery rescues neurological function at birth in sheep with spina bifida. Nat Med 1:342-347, 1995

10. Pedreira DA, Zanon N, Nishikuni K, Moreira de Sá RA, Acacio GL, Chmait RH, et al: Endoscopic surgery for the antenatal treatment of myelomeningocele: the CECAM trial. Am J Obstet Gynecol 214:111.e1-111.e11, 2016

11. Peranteau WH, Adzick NS: Prenatal surgery for myelomeningocele. Curr Opin Obstet Gynecol 28:111-118, 2016

12. Souka AP, Pilalis A, Kavalakis I, Antsaklis P, Papantoniou $\mathrm{N}$, Mesogitis S, et al: Screening for major structural abnormalities at the 11- to 14-week ultrasound scan. Am J Obstet Gynecol 194:393-396, 2006

13. Stiefel D, Copp AJ, Meuli M: Fetal spina bifida in a mouse model: loss of neural function in utero. J Neurosurg 106 (3 Suppl):213-221, 2007

14. Sutton LN: Fetal surgery for neural tube defects. Best Pract Res Clin Obstet Gynaecol 22:175-188, 2008

15. Tennant PW, Pearce MS, Bythell M, Rankin J: 20-year survival of children born with congenital anomalies: a population-based study. Lancet 375:649-656, 2010

16. Tulipan N, Wellons JC III, Thom EA, Gupta N, Sutton LN, Burrows PK, et al: Prenatal surgery for myelomeningocele and the need for cerebrospinal fluid shunt placement. J Neurosurg Pediatr 16:613-620, 2015

\section{Disclosures}

The authors report no conflict of interest concerning the materials or methods used in this study or the findings specified in this paper.

\section{Author Contributions}

Conception and design: Carrabba. Acquisition of data: Macchini, Fabietti, Boito, Porro, Gabetta, Picciolini, Ciralli. Analysis and interpretation of data: Cinnante, Triulzi. Drafting the article: Carrabba, Schisano, Meccariello. Critically revising the article: Campanella, Bertani, Locatelli. Study supervision: Mosca, Lapa, Leva, Rampini, Persico.

\section{Correspondence}

Giorgio Carrabba: Fondazione IRCCS Ca' Granda Ospedale Maggiore Policlinico, Milan, Italy. giorgio.carrabba@policlinico. mi.it. 